\title{
La médecine d'urgence de nouveau en première ligne face au risque émergent 2019-nCoV
}

\author{
Emergency Medicine Back on the Front Line against the Emerging Risk 2019-nCoV
}

\author{
P. Hausfater $\cdot$ N. Na $\cdot$ Y. Zhao \\ (C) SFMU et Lavoisier SAS 2020
}

L'actualité sanitaire de fin 2019 et début 2020 est dominée par la survenue d'une épidémie d'infection respiratoire due à un nouveau coronavirus 2019-nCoV, dont le foyer trouve son origine dans la ville de Wuhan, province du Hubei, en Chine centrale [1]. Près de 18 ans après l'épidémie de severe acute respiratory syndrom (SARS) et huit ans après celle du Middle East respiratory syndrom (MERS), les projecteurs semblent irrémédiablement devoir se tourner vers l'émergence d'un nouveau coronavirus à chaque décennie.

Les coronavirus sont des virus à ARN, endémiques chez l'homme, et dont les principales espèces $(\mathrm{hCoV}-229 \mathrm{E}$, OC43, NL63, HKU1) sont responsables d'infections respiratoires bénignes. Les épisodes de 2002 (SRAS), de 2012 (MERS) et maintenant de 2020 ( $\mathrm{n}-\mathrm{CoV}$ ) ont mis en lumière la capacité de certaines espèces de coronavirus de passer, à la faveur de modifications de protéines de surface, d'une espèce animale à l'homme [2]. S'agissant d'une nouvelle espèce pour laquelle l'homme n'est pas immunisé, la pathogénicité et le potentiel de contagiosité de ces nouveaux coronavirus sont majeurs à l'échelle planétaire, d'où les mesures exceptionnelles qui sont mises en place tant localement que sous l'égide de l'Organisation mondiale de la santé.

Tenant compte des enseignements des précédentes épidémies, la découverte du 2019-nCov s'est faite dans un temps record : à peine un mois après l'identification clinique d'un cluster de pneumonies sévères dans la ville de Wuhan, dues à un agent pathogène inconnu, le génome du 2019-nCov a été entièrement séquencé et partagé avec l'ensemble de la communauté scientifique internationale, permettant de construire des amorces pour les techniques de biologie moléculaire et donc le diagnostic positif en quelques heures à partir de pré-

\footnotetext{
P. Hausfater $(\bowtie) \cdot N$. Na

Service des urgences, hôpital Pitié-Salpêtrière, AP-HP,

Sorbonne Université, F-75013 Paris, France

e-mail : pierre.hausfater@aphp.fr

P. Hausfater

UMR Inserm 1166 IHU ICAN, GRC-14 BIOSFAST,

Sorbonne Université, F-75013 Paris, France
}

lèvements respiratoires [2]. Toutes aussi impressionnantes sont les mesures prises par les autorités chinoises pour tenter de contenir la diffusion épidémique de ce nouveau virus, avec notamment le confinement réel d'une ville (Wuhan) de 11 millions d'habitants !

Si à ce jour la mortalité liée au 2019-nCoV semble faible ( $2 \%$, comparée aux $10 \%$ du SARS et $34 \%$ du MERS), la vigilance est de règle : ce n'est pas tant le taux de mortalité que la perspective de diffusion à l'ensemble d'une population non protégée qui est à même de déstabiliser un système de santé [3]. Comme pour tout nouvel agent, même si de nombreuses similitudes existent avec les précédents coronavirus, des incertitudes persistent sur les modes de contamination, la contagiosité et la durée d'incubation, entre autres. La transmission interhumaine est d'ores et déjà prouvée, de même que la contamination nosocomiale, notamment au personnel soignant [4].

En France, la réponse à ce nouveau risque émergent a été immédiate et s'appuie également sur les expériences précédentes et surtout sur la médecine d'urgence et les services des maladies infectieuses et tropicales (MIT) des établissements de santé de référence (ESR). Les services d'urgence sont ainsi de nouveau en première ligne : mode d'accès aux soins non programmés et porte d'entrée de nombreux patients à l'hôpital, ouverts 24 heures/24, 365 jours par an (Fig. 1). Le rôle des structures d'urgence (services d'urgence et centres 15) est fondamental pour identifier et orienter les patients suspects et assurer le rôle de « gardien de but » afin d'éviter l'irruption involontaire d'un cas possible dans une salle d'attente ou une zone de soins bondées où le risque de diffusion serait majeur. Il n'aura d'ailleurs pas échappé que

N. Na

Consultation d'interface sino-française (CISF), hôpital Pitié-Salpêtrière, AP-HP, Sorbonne Université, F-75013 Paris, France

Y. Zhao

Service des urgences, hôpital Zhongnan, université de Wuhan, Wuhan, République populaire de Chine 
Nouveau CORONAVIRUS 新型冠状病毒: 2019-nCoV

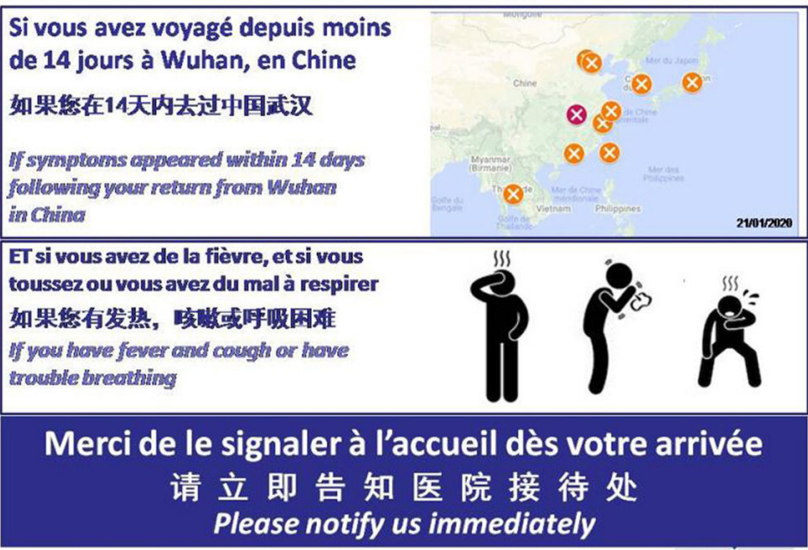

Fig. 1 Affiche présente à l'entrée du service d'urgence de l'hôpital Pitié-Salpêtrière. Document Assistance publique-Hôpitaux de Paris

ce risque émergent vient se télescoper avec l'épidémie annuelle de grippe ! S'agissant d'un mode de contamination principal de type " gouttelettes », les structures d'urgence ont acquis au fil du temps le réflexe de délivrer dès l'accueil un masque chirurgical à tout patient présentant de la fièvre et/ou des signes respiratoires. L'émergence de ce nouveau risque infectieux vient rappeler le caractère impératif et systématique de cette mesure, à toute période de l'année.

La médiatisation effrénée autour du 2019-nCov crée malheureusement un bruit de fond nuisible pour une prise en charge raisonnée du risque épidémique, avec son lot de fake news générant autant d'appels indus aux centres 15 ou de consultations inappropriées aux urgences. Face à ce désordre grandissant, la seule réponse est la rigueur scientifique des centres 15 et des services d'urgence pour, en lien avec les services de MIT des ESR, classer les cas suspects en exclu ou possible. À ce titre, les définitions de cas et de conduites à tenir publiées par Santé publique France sont suffisamment claires pour permettre aux médecins urgentistes de classer les patients suspects sur un simple interrogatoire [5]. Ces définitions sont cependant amenées à se modifier en fonction de l'évolution des connaissances sur ce virus, d'une part, et de l'ampleur de l'épidémie, d'autre part. Il n'est pas exclu que, dans l'hypothèse extrême d'une multiplication exponentielle des cas en France, des consignes de confinement au domicile soient appliquées, afin de ne pas saturer les établissements de santé.

Le trio centres 15-services d'urgence-infectiologues des ESR est un trio gagnant, qui a appris au fil du temps à travailler ensemble et à se répartir les rôles : régulation des appels et des patients pour les deux premiers, accueil des cas possibles par un vecteur régulé par le SAMU sans passer par un service d'urgence pour les services de MIT des ESR. Le devenir de l'épidémie en France est inconnu, mais au jour d'écriture de cet éditorial (2020), la France ne compte toujours que six patients confirmés et aucun décès. Efficacité des mesures de confinement en Chine ou excellence de la veille sanitaire en France, les prochaines semaines seront quoi qu'il en soit riches d'enseignement !

Liens d'intérêts : les auteurs déclarent ne pas avoir de liens d'intérêts.

\section{Références}

1. Zhu N, Zhang D, Wang W, et al (2020) A novel coronavirus from patients with pneumonia in China, 2019. N Engl J Med 382: [in press]

2. Wang C, Horby PW, Hayden FG, Gao GF (2020) A novel coronavirus outbreak of global health concern. Lancet 397: [in press]

3. Huang C, Wang Y, Li X, et al (2020) Clinical features of patients infected with 2019 novel coronavirus in Wuhan, China. Lancet 397: [in press]

4. Chan JFW, Yuan S, Kok KH, et al (2020) A familial cluster of pneumonia associated with the 2019 novel coronavirus indicating person-to-person transmission: a study of a family cluster. Lancet 397: [in press]

5. Santé publique France (2020) Cas de pneumonies associées à un nouveau coronavirus (2019-nCov) à Wuhan, en Chine. https:// www.santepubliquefrance.fr/maladies-et-traumatismes/maladies-etinfections-respiratoires/infection-a-coronavirus/articles/cas-depneumonies-associees-a-un-nouveau-coronavirus-2019-ncov-awuhan-en-chine. (Dernier accès le 28 janvier 2020) 